\title{
Velocity and energy distributions in microcanonical ensembles of hard spheres
}

\author{
Enrico Scalas, ${ }^{1,2, *}$ Adrian T. Gabriel, ${ }^{3, \dagger}$ Edgar Martin,,${ }^{3, \ddagger}$ and Guido Germano ${ }^{4,5, \S}$ \\ ${ }^{1}$ School of Mathematical and Physical Sciences, University of Sussex, Falmer, Brighton BN1 9RH, United Kingdom \\ ${ }^{2}$ Basque Center for Applied Mathematics, Alameda de Mazarredo 14, 48009 Bilbao, Basque Country, Spain \\ ${ }^{3}$ Department of Chemistry and WZMW, Philipps-University Marburg, 35032 Marburg, Germany \\ ${ }^{4}$ Department of Computer Science, University College London, Gower Street, London WC1E 6BT, United Kingdom \\ ${ }^{5}$ Systemic Risk Centre, London School of Economics and Political Science, Houghton Street, London WC2A 2AE, United Kingdom
}

(Received 27 August 2013; revised manuscript received 16 July 2015; published 25 August 2015)

\begin{abstract}
In a microcanonical ensemble (constant $N V E$, hard reflecting walls) and in a molecular dynamics ensemble (constant $N V E \mathbf{P G}$, periodic boundary conditions) with a number $N$ of smooth elastic hard spheres in a $d$ dimensional volume $V$ having a total energy $E$, a total momentum $\mathbf{P}$, and an overall center of mass position $\mathbf{G}$, the individual velocity components, velocity moduli, and energies have transformed beta distributions with different arguments and shape parameters depending on $d, N, E$, the boundary conditions, and possible symmetries in the initial conditions. This can be shown marginalizing the joint distribution of individual energies, which is a symmetric Dirichlet distribution. In the thermodynamic limit the beta distributions converge to gamma distributions with different arguments and shape or scale parameters, corresponding respectively to the Gaussian, i.e., MaxwellBoltzmann, Maxwell, and Boltzmann or Boltzmann-Gibbs distribution. These analytical results agree with molecular dynamics and Monte Carlo simulations with different numbers of hard disks or spheres and hard reflecting walls or periodic boundary conditions. The agreement is perfect with our Monte Carlo algorithm, which acts only on velocities independently of positions with the collision versor sampled uniformly on a unit half sphere in $d$ dimensions, while slight deviations appear with our molecular dynamics simulations for the smallest values of $N$.
\end{abstract}

DOI: 10.1103/PhysRevE.92.022140

\section{INTRODUCTION}

The problem of the velocity distribution in a gas of hard spheres was discussed in a paper published by Maxwell in 1860 [1]. Maxwell obtained the velocity distribution by assuming independence of the three components of velocity and rotational invariance of the joint distribution. The only distribution satisfying the functional equation

$$
\begin{aligned}
f_{\mathbf{v}}\left(x_{1}, x_{2}, x_{3}\right) & =\Phi\left(x_{1}^{2}+x_{2}^{2}+x_{3}^{2}\right) \\
& =f_{v_{1}}\left(x_{1}\right) f_{v_{2}}\left(x_{2}\right) f_{v_{3}}\left(x_{3}\right)
\end{aligned}
$$

has factors of the form

$$
f_{v_{\alpha}}(x)=A \exp \left(-B x^{2}\right),
$$

$\alpha=1,2,3$. This simple heuristic derivation can still be found in modern textbooks in statistical physics or physical chemistry [2], but generalizations of Maxwell's method appeared earlier in the physical literature [3].

In 1867, Maxwell [4] became aware that Eq. (2) should appear as a stationary solution for the dynamics of the gas and introduced the assumption of molecular chaos, according to which the velocities of two colliding molecules are uncorrelated and independent of their positions. This concept was later called Stoßzahlansatz or collision number hypothesis by Boltzmann. It led to a more detailed study of molecular collisions and to kinetic equations whose stationary solutions coincide with Maxwell's original distribution; see Refs. [5-9] for a modern mathematical approach to kinetic equations.

\footnotetext{
*e.scalas@sussex.ac.uk; www.sussex.ac.uk/profiles/330303

$\dagger$ adriangabriel@gmx.de

†emartin@gmx.de

§g.germano@ucl.ac.uk; www.cs.ucl.ac.uk/staff/g.germano
}

PACS number(s): 02.50.Ng, 05.10.Ln, 07.05.Tp

This route was followed by Boltzmann, who obtained the velocity distribution in a more general way in a series of papers published between 1868 and 1871 [10-12]. Based on the Stoßzahlansatz, Boltzmann could prove that Maxwell's distribution is stationary. These results are summarized in Tolman's book [13] and in the first chapter of ter Haar's book [14]. The Stoßzahlansatz provides an answer to Loschmidt's Umkehreinwand or reversibility paradox of 1876 [15], which questions how time-reversible microscopic dynamics can lead to time-irreversible results like the increase of the entropy of a gas as stated by Boltzmann's H-theorem of 1872 [16]: it was understood only later that the microscopic time reversibility of Newton's equations of motion was effectively destroyed by Boltzmann's use of the Stoßzahlansatz in his calculations. Boozer [17] provided a recent analysis of the role of the assumption of molecular chaos to obtain time-asymmetric results like Boltzmann's H-theorem and the Boltzmann transport equations by performing computer simulations of a one-dimensional system of two interlieved molecular species with mass ratios 1:2.

Boltzmann and Maxwell were not working in isolation and were aware of their respective works. In his 1872 paper, Boltzmann often quotes Maxwell [16]. In 1873, Maxwell wrote to his correspondent Tait [18]:

By the study of Boltzmann I have been unable to understand him. He could not understand me on account of my shortness, and his length was and is an equal stumbling block to me.

More details on the relationship between Maxwell and Boltzmann and on the influence of Maxwell on Boltzmann's thought have been collected by Uffink [19].

Tolman's analysis of classical binary collisions for hard spheres led to rate equations which can be interpreted as transition probabilities for a Markov chain after proper 
normalization. The interested reader can consult chapter V of Tolman's classic book [13], in particular the discussion around Eq. (45.3) on page 129. The connection with Markov chains was made explicit by Costantini and Garibaldi [20,21], who used a model due to Brillouin [22]. Before Costantini and Garibaldi, Penrose suggested that a Markovian hypothesis could justify the use of standard statistical mechanical tools [23]. According to our interpretation of Penrose, due to the limits in human knowledge naturally leading to coarse graining, systems of many interacting particles effectively behave as Markov chains. Moreover, the possible number of states of such a chain is finite even if very large, therefore only the theory of finite Markov chains is useful. Statistical equilibrium is reached when the system states obey the equilibrium distribution of the finite Markov chain; this equilibrium distribution exists, is unique, and coincides with the stationary distribution if the chain is irreducible and aperiodic. This point of view is also known as Markovianism. Indeed, in a recent paper on the Ehrenfest urn, or dogs and fleas model, we showed that, after appropriate coarse graining, a Markov chain well approximates the behavior of a realistic model for a fluid [24].

Here, we study the velocity distribution in a system of $N$ smooth elastic hard spheres in $d$ dimensions. Even if the evolution of the system is deterministic, we can consider the velocity components of each particle as random variables. We do not consider a finitary [25] version of the model by discretizing velocities, but keep them as real variables. Then a heuristic justification of Eq. (2) can be based on the central limit theorem (CLT). Here is the argument. Following Maxwell's idea, one can consider the velocity components of each particle independent from each other. Further assuming that velocity jumps after collisions are independent and identically distributed random variables, one obtains for the velocity component $\alpha$ of a particle $i$ at time $t$ :

$$
v_{i \alpha}(t)=v_{i \alpha}(0)+\sum_{j=1}^{n(t)} \Delta v_{i \alpha, j},
$$

where $n(t)$ is the number of collisions for that particle up to time $t$ and $\Delta v_{i \alpha, j}$ is the change in velocity at collision $j$. If the hypotheses stated above are valid, Eq. (3) defines a continuous-time random walk, and the distribution $f_{v_{i \alpha}}(x, t)$ approaches a normal distribution for large $t$ as a consequence of the CLT. Unfortunately this argument is only approximately true in the case of large systems and false for smaller systems.

In Sec. II we obtain the theoretical probability density functions of the individual energies, velocity moduli, and velocity components, starting from the fundamental uniform distribution law in phase space. In Sec. III we present the molecular dynamics method used to simulate hard spheres. Interestingly, the same distributions can be reproduced by a simple Monte Carlo stochastic model introduced in Sec. IV. The numerical results are presented in Sec. V together with some statistical goodness-of-fit tests. Indeed, it turns out that an equilibrium distribution of the velocity components seems to be reached already for $N=2$ particles and without using any coarse graining. When $N$ grows, the equilibrium distribution approaches the normal distribution, Eq. (2). A discussion and a summary follow in Sec. VI.

\section{THEORY}

We consider a fluid of $N$ hard spheres in $d$ dimensions with the same diameter $\sigma$ and mass $m$ in a cuboidal box with sides $L_{\alpha}, \alpha=1, \ldots, d$. The positions $\mathbf{r}_{i}, i=1, \ldots, N$, are confined to a $d$-dimensional box with volume $V=\prod_{\alpha=1}^{d} L_{\alpha}$; i.e., each position component $r_{i \alpha}$ can vary in the interval $\left[-L_{\alpha} / 2, L_{\alpha} / 2\right]$. Elastic collisions transfer kinetic energy between the particles, while the total energy of the system

$$
E=\frac{1}{2} \sum_{i=1}^{N} m_{i} v_{i}^{2}=\frac{1}{2} m v^{2}
$$

does not change in time; i.e., it is a constant of the motion. Therefore, the velocities $\mathbf{v}_{i}$ are confined to the surface of a hypersphere with radius $R=\sqrt{2 E / m}$ given by the constraint that the total energy is $E$; i.e., each velocity component $v_{i \alpha}$ can vary in the interval $[-R, R]$ with the restriction on the sum of the squares given by Eq. (4). In other words, the rescaled positions $\mathbf{q}$ with $q_{i \alpha}=r_{i \alpha} / L_{\alpha}$ are confined to the unit hypercube in $d N$ dimensions, while the rescaled velocity components $\mathbf{u}=\mathbf{v} / R$ are confined to the surface of the unit hypersphere in $d N$ dimensions defined by the constraint $u=\sqrt{\mathbf{u} \cdot \mathbf{u}}=1$.

The state of the system is specified by the phase space vector of all velocities and positions $\Gamma=(\mathbf{v}, \mathbf{r})$, i.e., by $2 d N$ variables: the velocity components $v_{i \alpha}$ and the position components $r_{i \alpha}$. However, these variables are not independent because of constraints. For spheres with random velocities and positions confined in a container with hard reflecting walls, the total energy $E$ is conserved and thus the degrees of freedom are $g=2 d N-1$; this is the microcanonical ensemble (constant $N V E$ ). Periodic boundary conditions conserve also the total linear momentum

$$
\mathbf{P}=\sum_{i=1}^{N} m_{i} \mathbf{v}_{i}=m \sum_{i=1}^{N} \mathbf{v}_{i}
$$

and the generator of Galilean transformations to other inertial frames of reference

$$
\mathbf{G}=\mathbf{P} t-\sum_{i=1}^{N} m_{i} \mathbf{r}_{i}=m \sum_{i=1}^{N}\left(\mathbf{v}_{i} t-\mathbf{r}_{i}\right),
$$

where the coordinates $\mathbf{r}_{i}$ are not reboxed upon a crossing of the unit cell boundaries (if $\mathbf{P}=\mathbf{0},-\mathbf{G} /\left(\sum_{i=1}^{N} m_{i}\right)=-\mathbf{G} /(\mathrm{Nm})$ is the position of the center of mass), and thus the number of independent variables drops to $g=2 d(N-1)-1=2 d N-$ $2 d-1$; this is the molecular dynamics ensemble (constant $N V E \mathbf{P G}$ ) [26-28]. Symmetries in the positions and velocities may reduce $g$ further; e.g. if all components $i$ of $\boldsymbol{\Gamma}$ are pairwise symmetric with respect to the origin, with both kinds of boundary conditions this point symmetry will stay on forever and $g=d N-1$ or $g=2 d(N / 2-1)-1=d N-2 d-1$, respectively. For the sake of simplicity, in presenting the theory we will treat explicitly only the microcanonical case without symmetries.

Following Khinchin [29], one can assume as the starting point of statistical mechanics that the distribution in the accessible portion of phase space is uniform, although so far this has not been rigorously proved in general. In our case, the 
measure of the accessible region of phase space is the product of the volume of the hypercube $V^{N}$ times the surface of the hypersphere with radius $R$ in $d N$ dimensions,

$$
\Omega=V^{N} \frac{2 \pi^{d N / 2}}{\Gamma(d N / 2)} R^{d N-1} .
$$

Then Khinchin's Ansatz that the probability density function (PDF) for points $(\mathbf{v}, \mathbf{r})$ in the permitted region of phase space is uniform leads to the joint PDF for velocities and positions

$$
f_{\mathbf{v}, \mathbf{r}}(\mathbf{x}, \mathbf{y})=\frac{1}{\Omega} \mathbf{1}_{\{\mathbf{x}: x=R\}}(\mathbf{x}) \mathbf{1}_{\left\{\mathbf{y}:-L_{\alpha} / 2 \leqslant y_{i \alpha} \leqslant L_{\alpha} / 2\right\}}(\mathbf{y}),
$$

where $\mathbf{1}_{A}(\mathbf{x})$ is the indicator function of the set $A$,

$$
\mathbf{1}_{A}(\mathbf{x}) \stackrel{\text { def }}{=}\left\{\begin{array}{lll}
1 & \text { if } & \mathbf{x} \in A \\
0 & \text { if } & \mathbf{x} \notin A
\end{array} .\right.
$$

As the energy does not depend on positions, one can integrate over the latter, yielding a uniform PDF for particle velocities on the surface of a hypersphere,

$$
f_{\mathbf{v}}(\mathbf{x})=\frac{\Gamma(d N / 2)}{2 \pi^{d N / 2}} R^{1-d N} \mathbf{1}_{\{\mathbf{x}: x=R\}}(\mathbf{x}) .
$$

The marginalization of this joint PDF leads to the distributions of individual particle energies as well as of velocity moduli and velocity components. To this purpose, it is convenient to study the relationship between Eq. (10) and the symmetric Dirichlet distribution with parameter $a$.

The PDF of the $n$-dimensional Dirichlet distribution with parameter vector $\mathbf{a}$ is

$$
f_{\mathbf{X}}^{\mathrm{D}}(\mathbf{x} ; \mathbf{a}) \stackrel{\text { def }}{=} \frac{1}{\mathrm{~B}(\mathbf{a})} \prod_{i=1}^{n} x_{i}^{a_{i}-1} \mathbf{1}_{S}(\mathbf{x}) ;
$$

it is zero outside the unit simplex

$$
S=\left\{\mathbf{x} \in \mathbb{R}^{n}: \forall i x_{i} \geqslant 0 \wedge \sum_{i=1}^{n} x_{i}=1\right\} .
$$

The normalization factor is the multinomial beta function, which can be defined through the gamma function,

$$
\mathrm{B}(\mathbf{a})=\frac{\prod_{i=1}^{n} \Gamma\left(a_{i}\right)}{\Gamma\left(\sum_{i=1}^{n} a_{i}\right)} .
$$

The multinomial beta function is a generalization of the beta function or Euler integral of the first kind, $\mathrm{B}(x, y)=$ $\int_{0}^{1} t^{x-1}(1-t)^{y-1} d t$, which can be expressed through the gamma function or Euler integral of the second kind, $\Gamma(z)=$ $\int_{0}^{\infty} e^{-t} t^{z-1} d t$, as $\mathrm{B}(x, y)=\Gamma(x) \Gamma(y) / \Gamma(x+y)$. In the symmetric Dirichlet distribution all elements of the parameter vector a have the same value $a_{i}=a$,

$$
f_{\mathbf{X}}^{\mathrm{D}}(\mathbf{x} ; a) \stackrel{\text { def }}{=} \frac{\Gamma(n a)}{[\Gamma(a)]^{n}} \prod_{i=1}^{n} x_{i}^{a-1} \mathbf{1}_{S}(\mathbf{x}) .
$$

Notice that $a=1$ gives the uniform distribution on $S$.

It is convenient to work with dimensionless variables. The rescaling $u_{i \alpha}=v_{i \alpha} / R$ introduced above gives the PDF

$$
f_{\mathbf{u}}(\mathbf{x})=\frac{\Gamma(d N / 2)}{2 \pi^{d N / 2}} \mathbf{1}_{\{\mathbf{x}: x=1\}}(\mathbf{x}) .
$$

A second transformation

$$
w_{i \alpha}=u_{i \alpha}^{2}
$$

leads to a set of $d N$ random variables each one with support in $[0,1]$ and such that

$$
\sum_{i=1}^{N} \sum_{\alpha=1}^{d} w_{i \alpha}=1 .
$$

The Jacobian for this transformation is

$$
\left|\frac{\partial \mathbf{u}}{\partial \mathbf{w}}\right|=\frac{1}{2^{d N}} \prod_{i=1}^{N} \prod_{\alpha=1}^{d} w_{i \alpha}^{-1 / 2} .
$$

Multiplying it by a factor $2^{d N}$ because each $\pm u_{i \alpha}$ results in the same $w_{i \alpha}$ and by another factor 2 because of the constraint given by Eq. (17) (for details see Song and Gupta [30]), and replacing $\sqrt{\pi}=\Gamma(1 / 2)$, the joint PDF of the variables $w_{i \alpha}$ can be expressed through the symmetric Dirichlet PDF with parameter $a=1 / 2$,

$$
\begin{aligned}
f_{\mathbf{w}}(\mathbf{x}) & =f_{\mathbf{w}}^{\mathrm{D}}(\mathbf{x} ; 1 / 2) \\
& =\frac{\Gamma(d N / 2)}{[\Gamma(1 / 2)]^{d N}} \prod_{i=1}^{N} \prod_{\alpha=1}^{d} x_{i \alpha}^{-1 / 2} \mathbf{1}_{S}(\mathbf{x}) .
\end{aligned}
$$

Now the normalized energy per particle

$$
\varepsilon_{i}=\frac{E_{i}}{E}=\frac{m v_{i}^{2}}{2 E}=\frac{v_{i}^{2}}{R^{2}}=\sum_{\alpha=1}^{d} w_{i \alpha}
$$

is the sum of $d$ variables distributed according to Eq. (19). As a consequence of the aggregation law for Dirichlet distributions, one finds that the joint PDF of all $\varepsilon_{i}$ is

$$
\begin{aligned}
f_{\boldsymbol{\varepsilon}}(\mathbf{x}) & =f_{\boldsymbol{\varepsilon}}^{\mathrm{D}}(\mathbf{x} ; d / 2) \\
& =\frac{\Gamma(d N / 2)}{[\Gamma(d / 2)]^{N}} \prod_{i=1}^{N} x_{i}^{d / 2-1} \mathbf{1}_{S}(\mathbf{x}) .
\end{aligned}
$$

It is interesting to notice that this is a uniform distribution for $d=2$; because of this, Boltzmann's 1868 method [10] works in $d=2$ dimensions, but fails for $d=3$.

The PDF of the normalized energies of single particles can be obtained by a further marginalization of the symmetric Dirichlet distribution given by Eq. (21), using again the aggregation law. The result is a beta distribution, whose PDF is

$$
f_{X}^{\beta}(x ; a, b) \stackrel{\text { def }}{=} \frac{1}{\mathrm{~B}(a, b)} x^{a-1}(1-x)^{b-1} \mathbf{1}_{[0,1]}(x),
$$

i.e., a Dirichlet distribution, Eq. (11), with $n=2$. In other words, the Dirichlet distribution is a multivariate generalization of the beta distribution. Our case has the exponents $a=d / 2$ and $b=d(N-1) / 2$,

$$
\begin{aligned}
f_{\varepsilon_{i}}(x) & =f_{\varepsilon_{i}}^{\beta}\left(x ; \frac{d}{2}, \frac{d(N-1)}{2}\right) \\
& =\frac{\Gamma(d N / 2)}{\Gamma(d / 2) \Gamma[d(N-1) / 2]} x^{\frac{d}{2}-1}(1-x)^{\frac{d(N-1)}{2}-1} \mathbf{1}_{[0,1]}(x) .
\end{aligned}
$$


The transformation of variables $E_{i}=E \varepsilon_{i}$ immediately leads to the beta-Stacy PDF of particle energies,

$$
\begin{aligned}
f_{E_{i}}(x)= & f_{E_{i}}^{\beta}\left(\frac{x}{E} ; \frac{d}{2}, \frac{d(N-1)}{2}\right) \frac{d}{d x} \frac{x}{E} \\
= & \frac{\Gamma(d N / 2)}{\Gamma(d / 2) \Gamma[d(N-1) / 2]}\left(\frac{x}{E}\right)^{\frac{d}{2}-1} \\
& \times\left(1-\frac{x}{E}\right)^{\frac{d(N-1)}{2}-1} \frac{1}{E} \mathbf{1}_{[0, E]}(x)
\end{aligned}
$$

for $N>1$, and $f_{E_{i}}(x)=\delta(x-E)$ for $N=1$. This result has been obtained with a different method, without invoking the Dirichlet and beta distributions, by Shirts et al. [28, Eq. (9)].

In the thermodynamic limit $(N, V, E \rightarrow \infty$ with $N / V=$ $\rho=$ constant and $E / N=\bar{E}=$ constant), Eq. (24) converges to a gamma distribution, as discussed by Garibaldi and Scalas [25, pp. 121-122]. The gamma PDF is

$$
f_{X}^{\gamma}(x ; a, b) \stackrel{\text { def }}{=} \frac{x^{a-1}}{b^{a} \Gamma(a)} \exp \left(-\frac{x}{b}\right) \mathbf{1}_{[0, \infty)}(x) .
$$

A scale parameter $b$ is usually included in the definition of the gamma distribution, but it can always be set to 1 absorbing it into the argument,

$$
f_{X}^{\gamma}(x ; a, b)=f_{X}^{\gamma}\left(\frac{x}{b} ; a, 1\right) \frac{1}{b} \equiv f_{X}^{\gamma}\left(\frac{x}{b} ; a\right) \frac{1}{b} .
$$

Coming back to the thermodynamic limit of Eq. (24) anticipated above, this is a gamma distribution with shape parameter $a=d / 2$ and scale parameter $b=2 \bar{E} /(d m)=R^{2} /(d N)$,

$$
\begin{aligned}
f_{E_{i}}(x) & =f_{E_{i}}^{\gamma}\left(x ; \frac{d}{2}, \frac{2 \bar{E}}{d m}\right) \\
& =\left(\frac{d m}{2 \bar{E}}\right)^{d / 2} \frac{x^{d / 2-1}}{\Gamma(d / 2)} \exp \left(-\frac{d m x}{2 \bar{E}}\right) \mathbf{1}_{[0, \infty)}(x),
\end{aligned}
$$

which is the familiar Boltzmann or Boltzmann-Gibbs distribution for $d=2$.

The PDF of the velocity moduli, or speeds, of individual particles can be obtained from $f_{E_{i}}(x)$ replacing $E_{i}=m v_{i}^{2} / 2$. The result is a transformed beta-Stacy distribution with the same exponents $a=d / 2$ and $b=d(N-1) / 2$ as for the energies, but argument $m x^{2} /(2 E)=(x / R)^{2}$,

$$
\begin{aligned}
f_{v_{i}}(x)= & f_{v_{i}}^{\beta}\left(\frac{x^{2}}{R^{2}} ; \frac{d}{2}, \frac{d(N-1)}{2}\right) \frac{d}{d x} \frac{x^{2}}{R^{2}}, \\
= & \frac{\Gamma(d N / 2)}{\Gamma(d / 2) \Gamma[d(N-1) / 2]} \frac{2 x^{d-1}}{R^{d}} \\
& \times\left(1-\frac{x^{2}}{R^{2}}\right)^{\frac{d(N-1)}{2}-1} \mathbf{1}_{[0, R]}(x)
\end{aligned}
$$

for $N>1$, and $f_{v_{i}}(x)=\delta(x-R)$ for $N=1$. Also this result has been obtained with a different method [28,31,32].

In the thermodynamic limit, Eq. (28) converges to a transformed gamma distribution with argument $x^{2} / 2$, shape parameter $a=d / 2$ and scale parameter

$$
\begin{aligned}
& b=R^{2} /(d N)=2 \bar{E} /(d m), \\
& f_{v_{i}}(x)=f_{v_{i}}^{\gamma}\left(\frac{x^{2}}{2} ; \frac{d}{2}, \frac{2 \bar{E}}{d m}\right) \frac{d}{d x} \frac{x^{2}}{2} \\
&=\left(\frac{d m}{\bar{E}}\right)^{\frac{d}{2}} \frac{(x / 2)^{d-1}}{\Gamma(d / 2)} \exp \left(-\frac{d m x^{2}}{4 \bar{E}}\right) \mathbf{1}_{[0, \infty)}(x),
\end{aligned}
$$

which is the familiar Maxwell distribution for $d=3$.

The transformation from hyperspherical to cartesian coordinates $v_{i}^{2}=\sum_{\alpha=1}^{d} v_{i \alpha}^{2}$ and $\left[2 \pi^{d / 2} / \Gamma(d / 2)\right] v_{i}^{d-1} d v_{i}=$ $\prod_{\alpha=1}^{d} d v_{i \alpha}$ leads from Eq. (28) to the PDF $f_{\mathbf{v}_{i}}(\mathbf{x})$ of the single-particle velocity vectors

$$
f_{\mathbf{v}_{i}}(\mathbf{x})=\frac{\Gamma(d N / 2)}{\Gamma[d(N-1) / 2]}\left(1-\frac{x^{2}}{R^{2}}\right)^{\frac{d(N-1)}{2}-1} \frac{\mathbf{1}_{\{\mathbf{x}: x=R\}}(\mathbf{x})}{(\sqrt{\pi} R)^{d}},
$$

an equation which has been obtained before too [28,31].

The direct marginalization [30] of the joint PDF of all velocities, Eq. (10), leads to the PDF $f_{v_{i \alpha}}(x)$ of velocity components, a result obtained integrating over all $i$ except one and over all $\alpha$ except one. This is the quantity discussed by Maxwell [1], and its derivation for any $N$ is one of the main results in this paper. It turns out that the distribution of the velocity components is a transformed beta with argument $1 / 2+x /(2 R)$ and equal exponents $a=b=(d N-1) / 2$,

$$
\begin{aligned}
f_{v_{i \alpha}}(x)= & \frac{1}{\mathrm{~B}((d N-1) / 2,(d N-1) / 2)} \\
& \times\left[\left(\frac{1}{2}+\frac{x}{2 R}\right)\left(\frac{1}{2}-\frac{x}{2 R}\right)\right]^{\frac{d N-3}{2}} \frac{\mathbf{1}_{[-R, R]}(x)}{2 R} \\
= & \frac{\Gamma(d N-1)}{\Gamma^{2}[(d N-1) / 2]}\left(1-\frac{x^{2}}{R^{2}}\right)^{\frac{d N-3}{2}} \frac{\mathbf{1}_{[-R, R]}(x)}{2^{d N-2} R} \\
= & f_{v_{i \alpha}}^{\beta}\left(\frac{1}{2}+\frac{x}{2 R} ; \frac{d N-1}{2}, \frac{d N-1}{2}\right) \\
& \times \frac{d}{d x}\left(\frac{1}{2}+\frac{x}{2 R}\right) .
\end{aligned}
$$

In the thermodynamic limit Eq. (31) converges to a Gaussian with average $\mu=0$ and variance $\sigma^{2}=R^{2} /(d N)=d \bar{E} /(2 m)$, i.e., the familiar Maxwell-Boltzmann distribution

$$
f_{v_{i \alpha}}(x)=\sqrt{\frac{m}{d \pi \bar{E}}} \exp \left(-\frac{m x^{2}}{d \bar{E}}\right) .
$$

This is again related to a gamma distribution, since the positive half of a Gaussian can be expressed as

$$
\frac{2}{\sqrt{2 \pi} \sigma} \exp \left(-\frac{x^{2}}{2 \sigma^{2}}\right) \mathbf{1}_{[0, \infty)}(x)=f_{X}^{\gamma}\left(\frac{x^{2}}{2} ; \frac{1}{2}, \sigma^{2}\right) \frac{d}{d x} \frac{x^{2}}{2} \text {. }
$$

In summary, all the known results for the relevant distributions of the $N V E$ ensemble can be obtained observing that the normalized individual particle energies $\varepsilon_{i}=E_{i} / E$ follow a symmetric multivariate Dirichlet distribution with parameter $a=1 / 2$ given by Eq. (19). This is a direct consequence of the uniform-distribution assumption in Eq. (8) via a simple change of variables. Only for the velocity components it is necessary to marginalize the uniform distribution directly on the surface 
of the hypersphere and not on the simplex. Maxwell's Ansatz is vindicated by the fact that, in the thermodynamic limit, a normal distribution for velocity components is recovered, as well as their independence. Finally, in the NVEPG ensemble the constraint given by Eq. (5) leads to similar distributions with different parameter values for the relevant quantities introduced above. This will become clearer in the following.

\section{MOLECULAR DYNAMICS SIMULATIONS}

In molecular dynamics (MD) with continuous potentials, the equations of motion are integrated numerically using a constant time step; this approach is called time-driven. The larger the forces, the smaller the time step necessary to ensure energy conservation. With step potentials there are no forces acting on a distance, only impulsive ones at the exact time of impact. Therefore an event-driven approach is more appropriate: rather than until a fixed time step, the system is propagated until either the next collision or the next boundary crossing [33-36].

The collision time $t_{i j}$ between two particles $i, j$ can be calculated from the mutual distance $\mathbf{r}_{i j}=\mathbf{r}_{i}-\mathbf{r}_{j}$ and the relative velocity $\mathbf{v}_{i j}=\mathbf{v}_{i}-\mathbf{v}_{j}$. If $b_{i j}=\mathbf{v}_{i j} \cdot \mathbf{r}_{i j}>0$ the particles are moving away from each other and will not collide. Otherwise impact may happen at time $t_{i j}$ when their distance becomes equal to the sum of their radii, i.e., $\left\|\mathbf{r}_{i j}+t_{i j} \mathbf{v}_{i j}\right\|=\sigma$. This is a second order problem with solutions

$$
t_{i j}^{ \pm}=\frac{-b_{i j} \pm \sqrt{b_{i j}^{2}-v_{i j}^{2}\left(r_{i j}^{2}-\sigma^{2}\right)}}{v_{i j}^{2}} .
$$

If the solutions are complex, no collision occurs. If the solutions are real, the smaller one, $t_{i j}^{-}$, corresponds to when the particles first meet, while the larger one, $t_{i j}^{+}$, to when they leave each other assuming they are allowed to interpenetrate. A negative collision time means that the event took place in the past. Because of the condition $b_{i j}<0$, at least $t_{i j}^{+}>0$. If $t_{i j}^{-}<0$ the particles overlap, which indicates an error. So the collision time is given by $t_{i j}^{-}$, provided it is a positive real number.

For a system of $N$ hard spheres, at impact, assuming an elastic collision, the total kinetic energy $E$ and the total linear momentum $\mathbf{P}$ are conserved (usually one sets $\mathbf{P}=\mathbf{0}$ at the beginning of the simulation by subtracting $\frac{1}{N} \sum_{i=1}^{N} \mathbf{v}_{i}$ from each $\mathbf{v}_{i}$ ). Assuming smooth surfaces, the impulse acts along the line of centers of the collision partners $i$ and $j$ given by the unit vector $\hat{\mathbf{r}}_{i j}=\mathbf{r}_{i j} / r_{i j}$; with equal masses, $\mathbf{v}_{i}$ changes to $\mathbf{v}_{i}+\Delta \mathbf{v}_{i}$ and $\mathbf{v}_{j}$ changes to $\mathbf{v}_{j}-\Delta \mathbf{v}_{i}$ with

$$
\Delta \mathbf{v}_{i}=-\frac{b_{i j} \mathbf{r}_{i j}}{\sigma^{2}}=-\left(\mathbf{v}_{i j} \cdot \hat{\mathbf{r}}_{i j}\right) \hat{\mathbf{r}}_{i j}=-\mathbf{v}_{i j}^{\|},
$$

where $\mathbf{r}_{i j}$ and $\mathbf{v}_{i j}$ are evaluated at the instant of collision, and thus $r_{i j}=\sigma$.

A few computational details for our event-driven molecular dynamics simulation of hard spheres are given in the Appendix.

\section{MONTE CARLO SIMULATIONS}

Except for especially ordered initial conditions, interparticle collisions computed by MD as explained in Sec. III have mutual distance versors at collision $\hat{\mathbf{r}}_{i j}$ uniformly distributed on a unit half sphere in $d$ dimensions such that, given relative velocities $\mathbf{v}_{i j}$, the scalar product $\mathbf{v}_{i j} \cdot \hat{\mathbf{r}}_{i j}$ is negative. Therefore the same distributions of velocities, and thus of derived quantities like energies, as in MD with periodic boundaries can be obtained by Monte Carlo (MC): after initializing the velocities of all hard spheres, the MC cycles consist in selecting a pair $i j$ and a random versor $\hat{\mathbf{r}}_{i j}$ such that $\mathbf{v}_{i j} \cdot \hat{\mathbf{r}}_{i j}<0$, and then in updating the velocities according to Eq. (35). Hard reflecting walls can be included in the MC scheme by selecting with a certain frequency a sphere $i$ and inverting one of its velocity components $v_{i \alpha}$. This scheme gives a useful insight into the mechanism of energy and momentum transfer. It is much easier to code and faster to run than MD, especially for large numbers of particles $N$, because no event list management is necessary: on the same computer used for the MD benchmarks shown in Fig. 3, the CPU time for $10^{5} \mathrm{MC}$ collisions with $N=10000$ spheres is $0.3 \mathrm{~s}$.

For a given initial state, i.e., a set of particle velocities, the MC dynamics defined above provides the realization of a Markov chain with a symmetric transition kernel, meaning that $P\left(\mathbf{v}^{\prime} \mid \mathbf{v}\right)=P\left(\mathbf{v} \mid \mathbf{v}^{\prime}\right)$, where $\mathbf{v}$ is the old velocity vector before the transition and $\mathbf{v}^{\prime}$ is the new velocity vector after the transition. This Markov chain is homogeneous, as the transition probability does not depend on the time step. Invoking detailed balance, $P\left(\mathbf{v}^{\prime} \mid \mathbf{v}\right) P(\mathbf{v})=P\left(\mathbf{v} \mid \mathbf{v}^{\prime}\right) P\left(\mathbf{v}^{\prime}\right)$, the symmetry of the transition kernel implies that the stationary distribution of this chain is uniform over the set of accessible states. If this set coincides with the surface of the velocity hypersphere, then the Markov chain is ergodic and one can hope to prove that the uniform distribution over the hypershere is also the equilibrium distribution for the Markov chain; see Sigurgeirsson [37, Chapter 5] for the discussion of a related problem and Meyn and Tweedie [38] for general methods. The results of MC simulations described below corroborate this conjecture and the algorithm outlined above is indeed an effective way of sampling the uniform distribution on the surface of a hypersphere.

\section{NUMERICAL RESULTS}

Probability density functions $f_{v_{i \alpha}}$ of the velocity components, $f_{v_{i}}$ of the velocity modulus, and $f_{E_{i}}$ of the energy for $N=2,3,4,10,100,1000$ hard disks $(d=2)$ and hard spheres $(d=3)$ from theory (Sec. II) as well as from MD (Sec. III) and MC simulations (Sec. IV) are shown in Fig. 1 for a microcanonical ensemble (constant $N V E$, hard reflecting walls) and in Fig. 2 for a molecular dynamics ensemble (constant $N V E \mathbf{P G}$, periodic boundary conditions). Simulations with $N=10000$ were done too but are not shown because the distributions overlap perfectly with those where $N=1000$. In reduced units [35] the particle mass is $m=1$, the particle diameter is $\sigma=1$, the energy per particle is $\bar{E}=1$, the Boltzmann constant is $k_{\mathrm{B}}=1$, and the number density is $\rho=2 / 3^{d}$. The density appears only in MD, and the results are largely independent of this parameter, as long as it is not too large (in this case the particles cannot move freely) or too small (in this case the particles hardly ever collide). The initial total momentum is $\mathbf{P}=\mathbf{0}$, except for $N=2$ with hard reflecting walls, and the initial position of the center of mass is in the origin. The numerical simulations were equilibrated over $5 \times 10^{5}$ collisions and sampled over $10^{6}$ collisions. 

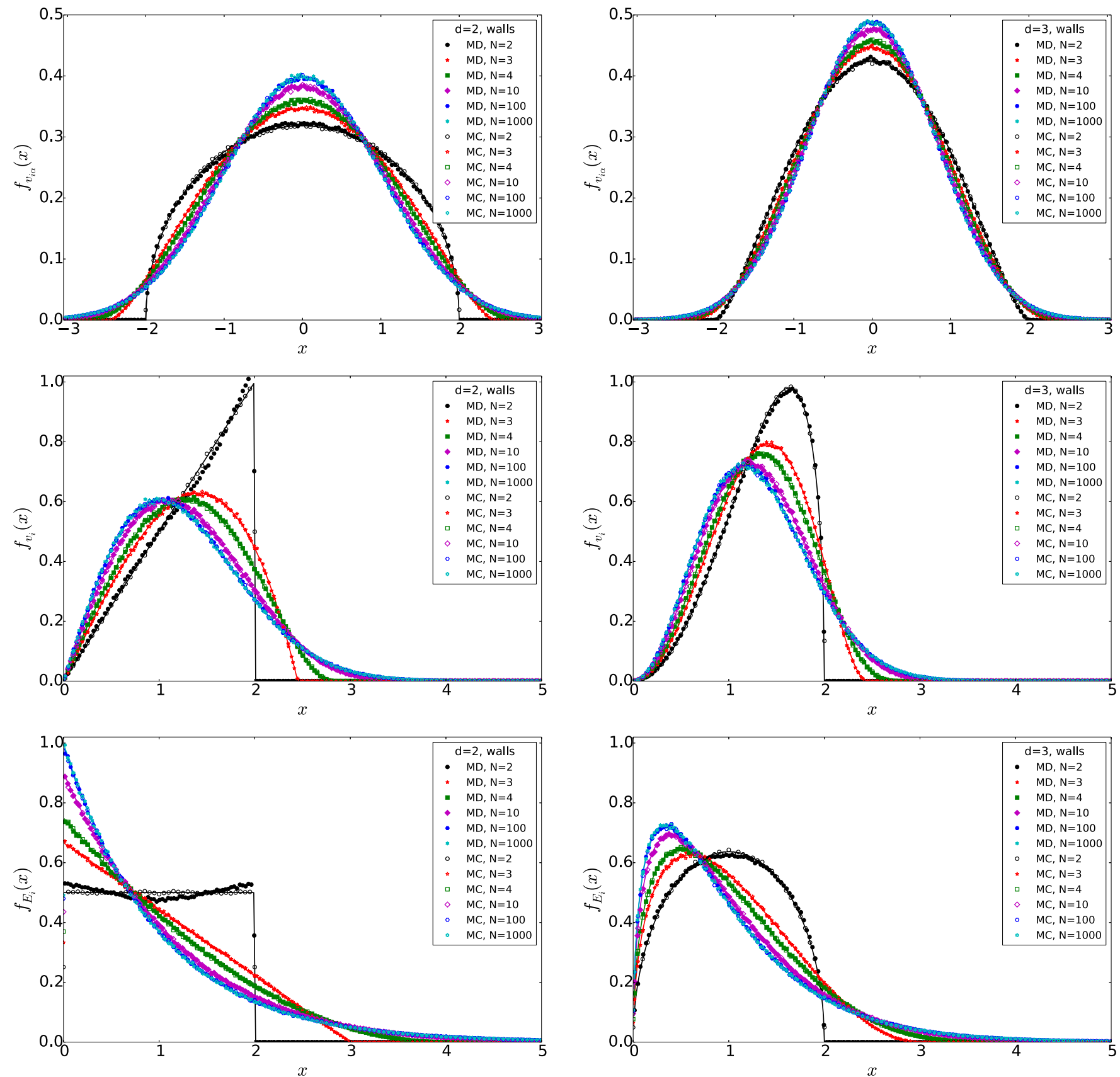

FIG. 1. (Color online) Probability density functions $f_{v_{i \alpha}}$ of the velocity components (top), $f_{v_{i}}$ of the velocity modulus (middle), and $f_{E_{i}}$ of the energy (bottom) for $d=2$ (left) and $d=3$ (right) with $\bar{E}=1$ in the microcanonical ensemble (constant $N V E$, hard reflecting walls): theory (lines), MD (full symbols), and MC (empty symbols).

We did not do the case with $d=1$ because then with equal masses Eq. (35) becomes $\Delta \mathbf{v}_{i}=-\mathbf{v}_{i j}^{\|}=-\mathbf{v}_{i j}$; thus after a collision $\mathbf{v}_{i}$ becomes $\mathbf{v}_{j}$ and vice versa, and with particles just exchanging their velocities, the velocity distribution does not change with time, making equilibration impossible. For this reason, one-dimensional models need systems of molecules with different masses [17].

The agreement between theory, MD, and MC is excellent, with little systematic deviations only for MD at the smallest values of $N$. To check that these deviations are genuine, we tried with different starting configurations and densities, and with both MD programs discussed in Sec. III, obtaining identical results. For further details on our MD simulations see Gabriel [39, Chapter 3].

For $d=2$ and $N=2$ in the $N V E \mathbf{P G}$ ensemble $f_{v_{i \alpha}}(x)$ is the arcsine PDF

$$
f_{v_{i \alpha}}(x)=\frac{1}{\pi \sqrt{R^{2}-x^{2}}},
$$

which is bimodal. The name is due to its cumulative distribution function

$$
F_{v_{i \alpha}}(x)=\frac{1}{\pi} \arcsin \frac{x}{R}+\frac{1}{2} .
$$



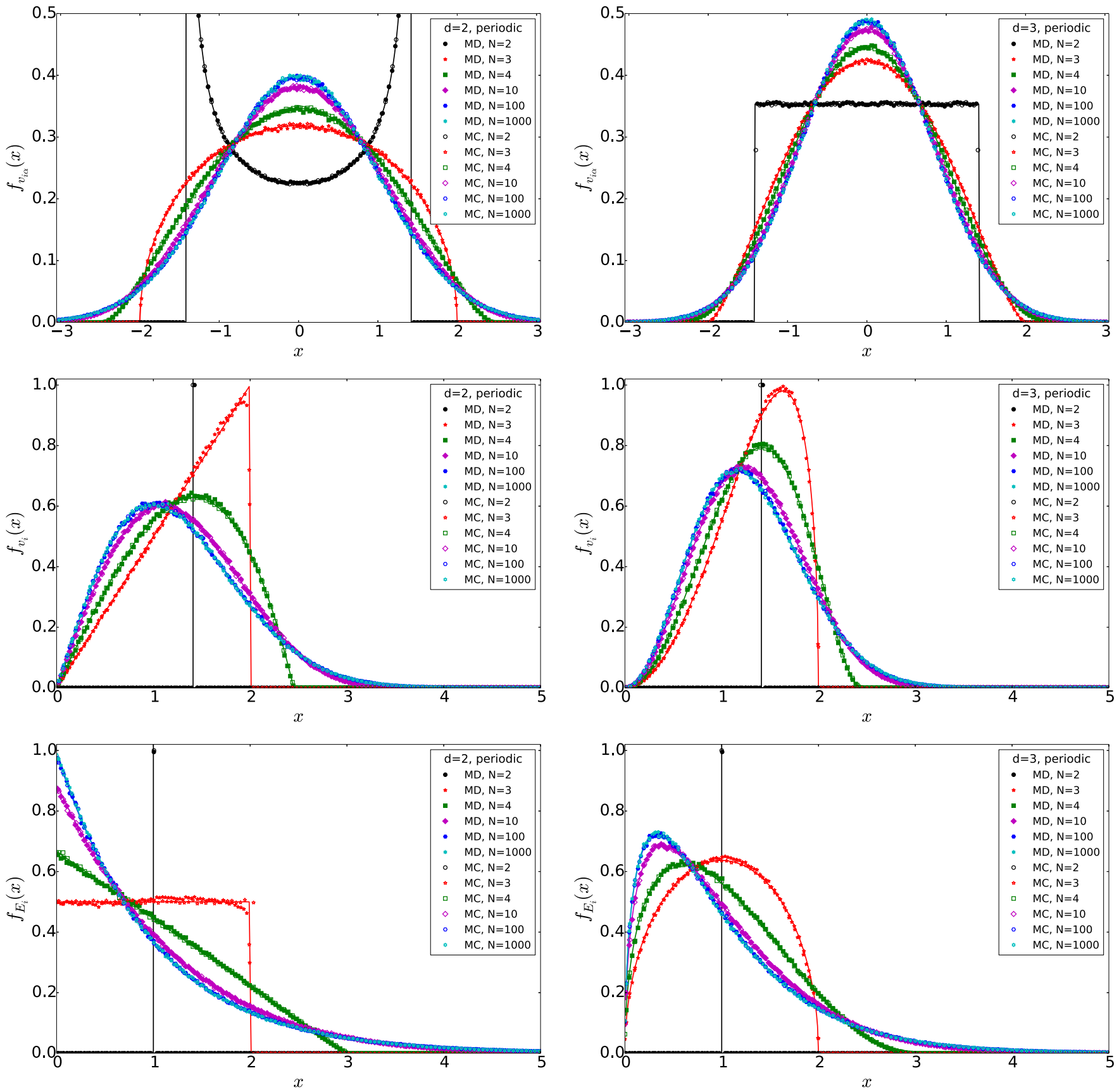

FIG. 2. (Color online) Probability density functions $f_{v_{i \alpha}}$ of the velocity components (top), $f_{v_{i}}$ of the velocity modulus (middle), and $f_{E_{i}}$ of the energy (bottom) for $d=2$ (left) and $d=3$ (right) with $\bar{E}=1$ in the molecular dynamics ensemble (constant $N V E \mathbf{P G}$, periodic boundary conditions): theory (lines), MD (full symbols), and MC (empty symbols). Delta functions are made visible by a vertical line for the theory and by rescaling down to 1 the data point that would otherwise be out of scale.

For $d=2$ and $N=2$ in the $N V E$ ensemble and for $d=2$ and $N=3$ in the $N V E \mathbf{P G}$ ensemble, $f_{v_{i \alpha}}(x)$ is the Wigner semicircle PDF [40]

$$
f_{v_{i \alpha}}(x)=\frac{2}{\pi R^{2}} \sqrt{R^{2}-x^{2}}
$$

Its cumulative distribution function again contains an arcsine:

$$
F_{v_{i \alpha}}(x)=\frac{x}{\pi R^{2}} \sqrt{R^{2}-x^{2}}+\frac{1}{\pi} \arcsin \frac{x}{R}+\frac{1}{2} .
$$

For the same systems, $f_{E_{i}}(x)$ is the uniform distribution on $[0, R]$. For $d=3$ and $N=2$ in the $N V E \mathbf{P G}$ ensemble, $f_{v_{i \alpha}}(x)$ is the uniform distribution on $[-\sqrt{R}, \sqrt{R}]$. All distributions are given by Eqs. (24), (28), and (31) inserting the appropriate values of $d, N$, and $E=N \bar{E}$ or $R=\sqrt{2 E / m}$ for the $N V E$ ensemble, while for the $N V E \mathbf{P G}$ ensemble $N$ must be substituted by $N-1$ because of the additional constraint on the linear momentum and thus on the center of mass.

To quantify the visual impression, in Table I we show Kolmogorov-Smirnov goodness-of-fit tests [41-43] 
TABLE I. Comparison between the empirical probability density functions of the velocity components from MC with periodic boundary conditions and $d=2$ by means of the Kolmogorov-Smirnov (KS) test. In each case the sample size is $2 \times 10^{6}$. At the $5 \%$ significance level the critical value is $9.6 \times 10^{-4}$. The null hypothesis of equally distributed data can never be rejected.

\begin{tabular}{lcc}
\hline \hline$N$ & Kolmogorov-Smirnov & $p_{\mathrm{KS}}$ \\
\hline 2 & $4.1 \times 10^{-4}$ & 0.99 \\
3 & $1.0 \times 10^{-3}$ & 0.24 \\
10 & $1.3 \times 10^{-3}$ & 0.07 \\
100 & $7.1 \times 10^{-4}$ & 0.69 \\
1000 & $6.2 \times 10^{-4}$ & 0.84 \\
10000 & $1.1 \times 10^{-3}$ & 0.15 \\
\hline \hline
\end{tabular}

comparing Eq. (31) with the empirical cumulative distribution function of the MC velocity components in the $N V E \mathbf{P G}$ ensemble for $d=2$ and $N=2,3,10,100,1000,10000$. In all cases the null hypothesis of data distributed according to the model equation cannot be rejected at the $5 \%$ significance level. The empirical density $f_{v_{i \alpha}}(x)$ is well approximated by a normal law for $N \geqslant 1000$ hard disks, as shown in Table II, where the results of two nonparametric tests for normality, Lilliefors [44] and Jarque and Bera [45,46], are presented for the MC velocity components of the systems with periodic boundary conditions, $d=2$ and $N=10,100,1000,10000$; these tests were made with the MATLAB functions lillietest and jbtest.

\section{DISCUSSION AND CONCLUSIONS}

To summarize what we have done, in a system of $N$ hard balls in a $d$-dimensional volume $V$ the velocity components, the velocity modulus and the energies of the spheres or disks are well reproduced by transformed beta distributions with different arguments and shape parameters depending on $N, d$, the total energy $E$, and the boundary conditions; in the thermodynamic limit these distributions converge to transformed gamma distributions with different arguments and shape or scale parameters, corresponding respectively to the Gaussian, i.e., Maxwell-Boltzmann, the Maxwell, and the Boltzmann or Boltzmann-Gibbs distribution. We showed this theoretically using Khinchin's Ansatz and performed statistical goodness-of-fit tests on systematic MD and MC computer simulations of an increasing number $N$ of hard disks or spheres

TABLE II. Results of two nonparametric normality tests for the empirical probability density function of the velocity components from MC with periodic boundary conditions when $d=2$ : Lilliefors (L) and Jarque and Bera (JB). In each case the sample size is $2 \times 10^{6}$. At the $5 \%$ significance level the critical value is $6.43 \times 10^{-4}$ for the $\mathrm{L}$ test and 5.99 for the JB test. The star indicates that the null hypothesis of normally distributed data can be rejected.

\begin{tabular}{lcccc}
\hline \hline$N$ & Lilliefors & $p_{\mathrm{L}}$ & Jarque-Bera & $p_{\mathrm{JB}}$ \\
\hline 10 & $0.008^{*}$ & $<10^{-3}$ & $7.4 \times 10^{3 *}$ & $<10^{-3}$ \\
100 & $7.36 \times 10^{-4 *}$ & 0.01 & $29.6^{*}$ & $<10^{-3}$ \\
1000 & $4.79 \times 10^{-4}$ & 0.35 & 5.70 & 0.06 \\
10000 & $4.37 \times 10^{-4}$ & 0.50 & 2.36 & 0.31 \\
\hline \hline
\end{tabular}

starting from 2 in the microcanonical ensemble (constant $N V E$, hard reflecting walls) and in the molecular dynamics ensemble (constant $N V E \mathbf{P G}$, periodic boundary conditions). The MC simulations are a simple stochastic model based on a generalization of Eq. (3) able to reproduce the same empirical equilibrium distribution for the random variables $v_{i \alpha}, v_{i}$, and $E_{i}$ as obtained deterministically with canonic dynamics by MD simulations. While the MC results overlap perfectly with theory, there is a slight disagreement of the MD results for the smallest values of $N$. A definitive explanation of this will require further investigation. However, one can recall that our MC scheme acts only on velocities independently of positions and the volume $V$, with the interparticle versor at collision sampled uniformly on a unit half sphere in $d$ dimensions. On the contrary, due to the canonical dynamics, in MD velocities and positions are mutually dependent; therefore geometrical constraints in the smallest systems may lead to a non perfectly uniform sampling of the state space. Moreover, in the MC systems with walls the correlation among velocities at impact is below 0.001 for every value of $N$ (random variables uniformly distributed on the hypersphere are uncorrelated even if dependent), so that the assumptions of molecular chaos are always fulfilled, whereas a computer simulation of a one-dimensional system has shown that in MD this happens only with growing $N$ [17].

We presented comprehensively both analytical derivations with a new approach and numerical checks, the latter both by MD and MC with a systematic investigation of parameter values and boundary conditions; we obtained also the PDF of velocity components, Eq. (31), which was not possible with previous approaches; we realized that all these distributions are variants of the beta or the gamma distribution and can be derived from the Dirichlet distribution; we pointed out that for values of $N$ as low as 2 or 3 the shapes of these distributions can be quite different from those in the thermodynamic limit: in particular, they can become uniform or even bimodal; last, we discussed the slight deviations of the MD results from theory.

The significance of our investigations goes beyond the foundations of statistical mechanics: few-body systems and microclusters are of current practical interest in applied fields such as nanotechnology and biophysics, and there is an increasing effort to understand the statistical mechanics of such systems, which departs from the traditional approach in the thermodynamic limit [47]. Another application of our results may be in the theory of thermostats or heat baths with a finite thermal capacity [48].

The MD simulations presented above corroborate Boltzmann's ergodic hypothesis [49] for both the $N V E$ and the $N V E \mathbf{P G}$ ensembles. Sinai [50] updated this hypothesis translating it in modern mathematical terms. One should prove that every hard-ball system on a flat torus, after fixing its total energy, momentum, and center of mass, is fully hyperbolic and ergodic; hyperbolic means that its Lyapunov exponent is nonzero almost everywhere with respect to the Liouville measure. This rephrasing of Boltzmann's hypothesis is known as the Boltzmann-Sinai ergodic hypothesis. The proofs of ergodicity for similar systems use the so-called Chernov-Sinai Ansatz, namely, the almost sure hyperbolicity of singular orbits [51]. More recently, after proving the ergodiciy of hard disks [52], Simányi published a proof of the Boltzmann-Sinai ergodic hypothesis in full generality for hard-ball systems [53]. 
MD simulations show that Khinchin's Ansatz is justified for systems of hard balls. We would like to stress that this is a consequence of the microscopic dynamics and not of any a priori maximum-entropy principle. The uniform distribution on the accessible phase-space region is indeed the maximumentropy distribution. Therefore, maximum-entropy methods do work well and all the distributions in Sec. II could be obtained by maximum-entropy methods: the beta and gamma distributions are actually the maximum-entropy distributions with given first moment, and possibly some other constraint, on a finite and a semi-infinite interval respectively. However, this is so only because the dynamics uniformly samples the accessible phase-space region and not the other way round. In different frameworks, e.g., in biology or economics, maximum-entropy assumptions might lead to wrong results for the equilibrium distribution of a system, if its dynamics is not specified or carefully studied.

The distributions derived in Sec. II are a benchmark for random partition models popular in econophysics. Pure exchange models often lead to the same distributions [25,54-59].

\section{ACKNOWLEDGMENTS}

We are thankful to Ubaldo Garibaldi for pointing us to the Dirichlet distribution as the multivariate generalization of the beta distribution, and to Mauro Politi for observing that the beta distribution maximizes the entropy on a finite interval given two constraints that include the first moment, even if we did not use this property in the theory exposed in Sec. II. This paper was partially supported by Italian MIUR grant PRIN 2009 H8WPX5 "The growth of firms and countries: distributional properties and economic determinants-Finitary and non-finitary probabilistic models in economics." The support of the UK Economic and Social Research Council (ESRC) in funding the Systemic Risk Centre is gratefully acknowledged (Grant No. ES/K002309/1).

\section{APPENDIX: COMPUTATIONAL DETAILS OF THE MOLECULAR DYNAMICS SIMULATIONS}

When a particle reaches a side of the unit box, periodic boundary conditions may require to "rebox" it by reintroducing it on the other side, while hard reflecting walls require to invert the velocity component perpendicular to the wall. After an event, be it a collision with another particle, a boundary crossing, or a reflection at a boundary, the event calendar must be reevaluated for pairs involving one of the event participants or a particle scheduled to collide with one of the event participants. All other particles are not influenced. Thus not every scheduled event actually takes place, because it can be invalidated by another earlier event, in which case it is erased from the priority queue. The latter is most commonly handled by means of a binary tree [60], which we realized with a multimap of the $\mathrm{C}++$ Standard Template Library [61].
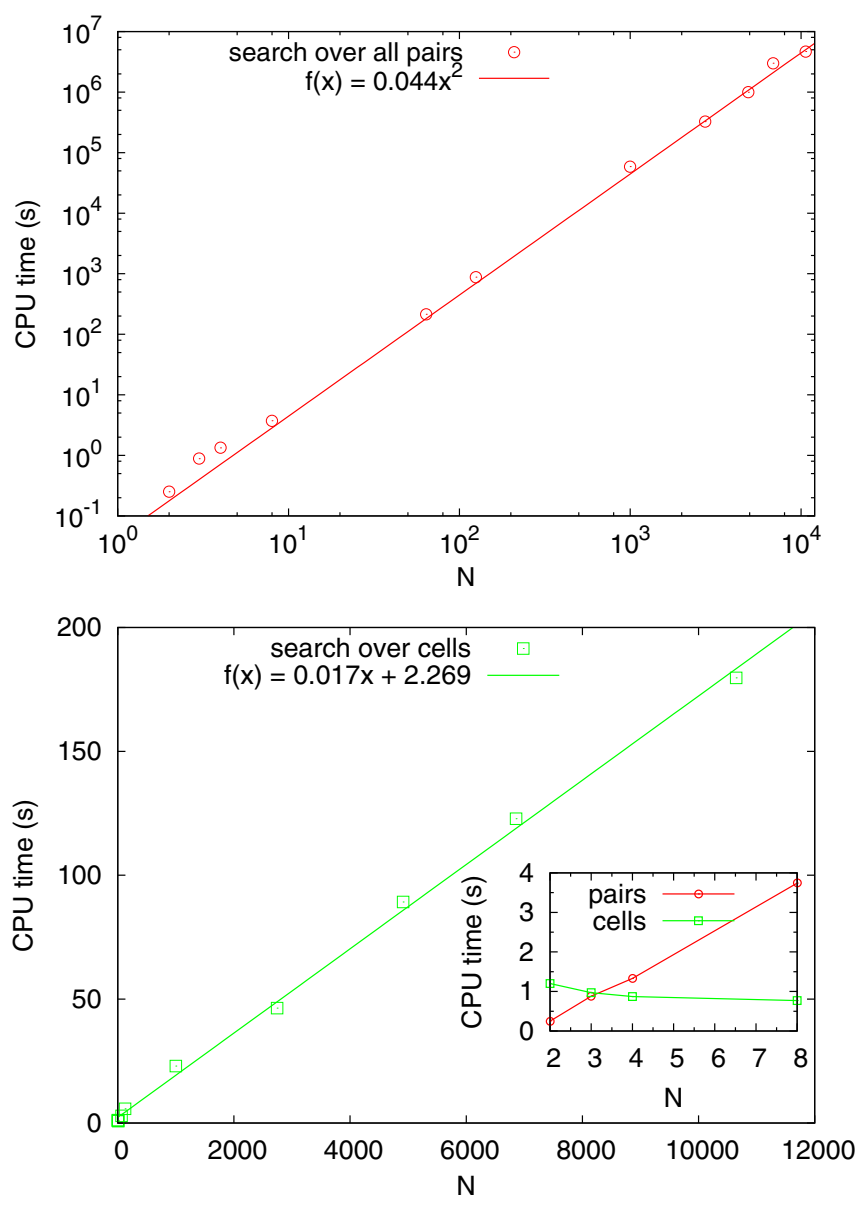

FIG. 3. (Color online) CPU time for $10^{5}$ collisions on a $2 \mathrm{GHz}$ Intel Core2 Duo as a function of the number of hard spheres $N$ for our two event-based MD programs, one with a simple search over all pairs (top), the other with an optimized search over cells (bottom). The data are fitted, respectively, by a quadratic and a linear function, which crossover between $N=3$ and $N=4$ (inset). The CPU times for the search over all pairs with $N \geqslant 1000$ were extrapolated from the CPU times for 100 collisions.

The efficiency of this and alternative data structures for event scheduling has been analyzed extensively $[62,63]$.

The computational effort to search for $\min _{i, j} t_{i j}$ grows as the square of the number of particles; see Fig. 3 (top). For large systems it is advisable to divide the simulation box into cells [26], which makes the dependence of the CPU time on the number of particles linear; see Fig. 3 (bottom). Provided cell boundary crossings are considered too in the event list, two particles can collide only if they are located in the same cell or in adjacent cells. We chose cells with a side larger than a particle diameter. For more details on this and other algorithmic aspects in event-driven MD see Refs. [64-66]. For a parallel implementation see Miller and Luding [67].
[1] J. C. Maxwell, Illustrations of the dynamical theory of gases. Part 1. On the motions and collisions of perfectly elastic spheres, Phil. Mag. 19, 19 (1860).

[2] P. W. Atkins, Physical Chemistry (Oxford University Press, Oxford, 1978).
[3] I. Maizlish, Maxwell's law of distribution of velocities and the principle of projective covariance, Phys. Rev. 20, 39 (1922).

[4] J. C. Maxwell, On the dynamical theory of gases, Phil. Trans. R. Soc. London 157, 49 (1860). 
[5] L. Desvillettes, C. Graham, and S. Méléard, Probabilistic interpretation and numerical approximation of a Kac equation without cut-off, Stoch. Proc. Appl. 84, 115 (1999).

[6] C. Graham and S. Méléard, Existence and regularity of a solution of a Kac equation without cut-off using the stochastic calculus of variations, Commun. Math. Phys. 205, 551 (1999).

[7] N. Fournier and S. Méléard, Monte Carlo approximations and fluctuations for 2D Boltzmann equations without cutoff, Markov Process. Relat. Fields 7, 159 (2001).

[8] N. Fournier and S. Méléard, Monte Carlo approximations for 2D Boltzmann equations without cutoff and for non Maxwell molecules, Monte Carlo Methods Appl. 7, 177 (2001).

[9] N. Fournier and S. Méléard, A Markov process associated with a Boltzmann equation without cutoff and for non Maxwell molecules, J. Stat. Phys. 104, 359 (2001).

[10] L. Boltzmann, Studien über das Gleichgewicht der lebendigen Kraft zwischen bewegten materiellen Punkten, Wien. Ber. 58, 517 (1868).

[11] L. Boltzmann, Über das Wärmegleichgewicht der lebendigen Kraft zwischen mehratomigen Gasmolekülen, Wien. Ber. 63, 397 (1871).

[12] L. Boltzmann, Einige allgemeine Sätze über Wärmegleichgewicht, Wien. Ber. 63, 679 (1871).

[13] R. C. Tolman, The Principles of Statistical Mechanics (Oxford University Press, Oxford, 1938).

[14] D. ter Haar, Elements of Statistical Mechanics (Rinehart \& Company, New York, 1954).

[15] J. Loschmidt, Über das Wärmegleichgewicht eines Systems von Körpern mit Rücksicht auf die Schwere, Wien. Ber. 73, 139 (1876).

[16] L. Boltzmann, Weitere Studien über das Wärmegleichgewicht unter Gasmolekülen, Wien. Ber. 66, 275 (1872).

[17] A. D. Boozer, Boltzmann's $H$-theorem and the assumption of molecular chaos, Eur. J. Phys. 32, 1391 (2011).

[18] J. C. Maxwell, Letter to P. G. Tait, August, 1873, in Maxwell on Heat and Statistical Mechanics, edited by E. Garber, S. G. Brush, and C. W. F. Everitt (Cambridge University Press, Cambridge, 1995), p. 123.

[19] J. Uffink, Boltzmann's work in statistical physics, in Stanford Encyclopedia of Philosophy, edited by E. N. Zalta (Stanford University, Stanford, 2008), http://plato.stanford.edu/entries/ statphys-Boltzmann.

[20] D. Costantini and U. Garibaldi, A probabilistic foundation of elementary particle statistics. Part I, Stud. Hist. Phil. Mod. Phys. 28, 483 (1997).

[21] D. Costantini and U. Garibaldi, A probabilistic foundation of elementary particle statistics. Part II, Stud. Hist. Phil. Mod. Phys. 29, 37 (1998).

[22] L. Brillouin, Comparison des différent statistiques appliquées aux problèmes des quanta, Ann. Phys.-Paris 7, 315 (1927).

[23] O. Penrose, Foundations of Statistical Mechanics (Pergamon Press, Oxford, 1970).

[24] E. Scalas, E. Martin, and G. Germano, Ehrenfest urn revisited: Playing the game on a realistic fluid model, Phys. Rev. E 76, 011104 (2007).

[25] U. Garibaldi and E. Scalas, Finitary Probabilistic Methods in Econophysics (Cambridge University Press, Cambridge, 2010).

[26] J. J. Erpenbeck and W. W. Wood, Molecular dynamics techniques for hard core systems, in Statistical Mechanics B. Modern
Theoretical Chemistry, edited by B. J. Berne (Plenum Press, New York, 1977), Vol. 6, pp. 1-40.

[27] J. R. Ray and H. Zhang, Phys. Rev. E 59, 4781 (1999).

[28] R. B. Shirts, S. R. Burt, and A. M. Johnson, Periodic boundary induced breakdown of the equipartition principle and other kinetic effects of finite sample size in classical hard-sphere molecular dynamics simulation, J. Chem. Phys. 125, 164102 (2006).

[29] A. I. Khinchin, Mathematical Foundations of Statistical Mechanics (Dover, New York, 1949).

[30] D. Song and A. K. Gupta, $L_{p}$-norm uniform distribution, P. Am. Math. Soc. 125, 595 (1997).

[31] M. J. Uline, D. W. Siderius, and D. S. Corti, On the generalized equipartition theorem in molecular dynamics ensembles and the microcanonical thermodynamics of small systems, J. Chem. Phys. 128, 124301 (2008).

[32] A. D. Boozer, The momentum distributions of a onedimensional ideal gas of $N$ atoms, Am. J. Phys. 78, 20 (2010).

[33] B. J. Alder and T. E. Wainwright, Phase transition for a hard sphere system, J. Chem. Phys. 27, 1208 (1957).

[34] B. J. Alder and T. E. Wainwright, Studies in molecular dynamics. I. General method, J. Chem. Phys. 31, 459 (1959).

[35] M. P. Allen and D. J. Tildesley, Computer Simulation of Liquids (Oxford University Press, Oxford, 1989).

[36] D. C. Rapaport, The Art of Molecular Dynamics Simulation, 2nd ed. (Cambridge University Press, Cambridge, 2004).

[37] H. Sigurgeirsson, Particle field models: Algorithms and applications, Ph.D. thesis, Scientific Computing and Computational Mathematics Program, Stanford University, 2002, http://citeseerx.ist.psu.edu/viewdoc/download?doi=10.1.1.85. $9447 \&$ rep $=$ rep $1 \&$ type $=$ pdf

[38] S. P. Meyn and R. L. Tweedie, Markov Chains and Stochastic Stability (Springer, London, 1993).

[39] A. T. Gabriel, Molekulardynamik und Molekulargraphik von Modellflüssigkeiten, Ph.D. thesis, Computer Simulation Group, Department of Chemistry, Philipps-University Marburg, 2010, http://archiv.ub.uni-marburg.de/diss/z2011/0055.

[40] M. Politi, E. Scalas, D. Fulger, and G. Germano, Spectral densities of Wishart-Lévy free stable random matrices, Eur. Phys. J. B 73, 13 (2010).

[41] A. N. Kolmogorov, Sulla determinazione empirica di una legge di distribuzione, Giornale dell' Istituto Italiano degli Attuari 4, 1 (1933).

[42] N. Smirnov, Sur les écarts de la courbe de distribution empirique, Recueil Mathématique (Mat. Sb.) 6(48), 3 (1939).

[43] F. J. Massey, The Kolmogorov-Smirnov test for goodness of fit, J. Am. Stat. Assoc. 46, 68 (1951).

[44] H. W. Lilliefors, On the Kolmogorov-Smirnov test for normality with mean and variance unknown, J. Am. Stat. Assoc. 62, 399 (1967).

[45] C. M. Jarque and A. K. Bera, Efficient tests for normality, homoscedasticity and serial independence of regression residuals, Econ. Lett. 6, 255 (1980).

[46] C. M. Jarque and A. K. Bera, Efficient tests for normality, homoscedasticity and serial independence of regression residuals: Monte Carlo evidence, Econ. Lett. 7, 313 (1981).

[47] T. Niiyama, Y. Shimizu, T. R. Kobayashi, T. Okushima, and K. S. Ikeda, Effect of translational and angular momentum conservation on energy equipartition in microcanonical equilibrium in small clusters, Phys. Rev. E 79, 051101 (2009). 
[48] M. Campisi, P. Talkner, and P. Hänggi, Finite bath fluctuation theorem, Phys. Rev. E 80, 031145 (2009).

[49] D. Szász, Boltzmann's ergodic hypothesis: A conjecture for centuries?, Studia Sci. Math. Hung. 31, 201 (1999).

[50] Y. G. Sinai, Ergodicity of Boltzmann's gas model, in Statistical Mechanics: Foundations and Applications, edited by T. A. Bak (W. A. Benjamin, New York, 1967), pp. 559-572.

[51] Y. Sinai and N. I. Chernov, Ergodic properties of some systems of 2-dimensional discs and 3-dimensional spheres, Russ. Math. Surv. 42, 181 (1987).

[52] N. Simányi, Proof of the Boltzmann-Sinai ergodic hypothesis for typical hard disk systems, Invent. Math. 154, 123 (2003).

[53] N. Simányi, The Boltzmann-Sinai ergodic hypothesis in full generality, arXiv:1007.1206.

[54] M. Patriarca, A. Chakraborti, K. Kaski, and G. Germano, Kinetic theory models for the distribution of wealth: Power law from overlap of exponentials, in Econophysics of Wealth Distributions, edited by A. Chatterjee, S. Yarlagadda, and B. K. Chakrabarti (Springer, Milan, 2005), pp. 93-110, series New Economic Windows, Vol. 2, arXiv:physics/0504153.

[55] M. Patriarca, A. Chakraborti, and G. Germano, Influence of saving propensity on the power-law tail of the wealth distribution, Physica A 369, 723 (2006).

[56] E. Scalas, U. Garibaldi, and S. Donadio, Statistical equilibrium in simple exchange games I, Eur. Phys. J. B 53, 267 (2006).

[57] U. Garibaldi, E. Scalas, and P. Viarengo, Statistical equilibrium in simple exchange games II. The redistribution game, Eur. Phys. J. B 60, 241 (2007).
[58] D. Matthes and G. Toscani, On steady distributions of kinetic models of conservative economies, J. Stat. Phys. 130, 1087 (2008).

[59] A. Chakraborti and G. Germano, Agent-based models of economic interactions, in Mathematical Modeling of Collective Behavior in Socio-Economic and Life Sciences, edited by G. Naldi, L. Pareschi, and G. Toscani (Birkhäuser, Boston, 2010), pp. 3-29, series Modeling and Simulation in Science, Engineering and Technology, Vol. 31.

[60] D. C. Rapaport, Event scheduling problem in molecular dynamic simulation, J. Comput. Phys. 34, 184 (1980).

[61] N. M. Josuttis, The C++ Standard Library-A Tutorial and Reference (Addison-Wesley, Boston, 1999).

[62] M. Marin and P. Cordero, An empirical assessment of priorityqueues in event driven molecular dynamics simulation, Comput. Phys. Commun. 92, 214 (1995).

[63] G. Paul, A complexity $\mathrm{O}(1)$ priority queue for event driven molecular dynamics simulations, J. Comput. Phys. 221, 615 (2007).

[64] B. D. Lubachevsky, How to simulate billiards and similar systems, J. Comput. Phys. 94, 255 (1991).

[65] M. Marin, D. Risso, and P. Cordero, Efficient algorithms for many-body hard particle molecular dynamics, J. Comput. Phys. 109, 306 (1993).

[66] M. Isobe, Simple and efficient algorithm for large scale molecular dynamics simulation in hard disk system, Int. J. Mod. Phys. C 10, 1281 (1999).

[67] S. Miller and S. Luding, Event-driven molecular dynamics in parallel, J. Comput. Phys. 193, 306 (2003). 